- This study examines the relationship between enamel acid etch pattern and bond strength.

- Bond strength and etch patterns vary between tooth type and between teeth within a tooth type.

- An 'ideal' enamel etch pattern is not essential for high bond strength.

\title{
Relationship between enamel etch characteristics and resin-enamel bond strength
}

\author{
R. S. Hobson ${ }^{1}$ and J. F. McCabe ${ }^{2}$
}

\begin{abstract}
Objectives: The aim of the study was to investigate the relationship between etch patterns produced on surface enamel by phosphoric acid and the corresponding bond strengths achieved.

Materials and methods: Twenty-eight patients had the buccal surface of their teeth etched and replicated for examination under the scanning electron microscope, at the commencement of orthodontic treatment. Histometry was employed to assess the nature and quality of etch patterns using a four-point scale. Twenty of each tooth type (total of 240), were collected and A Company pre-adjusted orthodontic brackets, bonded using Transbond light-cured orthodontic adhesive according to the manufacturer's instructions and 24-hour bond strength testing was undertaken. The Adhesive Remnant Index (ARI) was recorded for the tooth and bracket surface for all specimens.

Results: The type of etch was not significantly affected by the side (left or right) of the mouth assessed ( $p=0.532)$. Overall, there was no significant difference between etch types for upper and lower teeth $(p=0.218)$; however, there were significant differences between specific teeth in the upper and lower arch. The greatest amount of Type A etch (well-defined etched enamel prisms) was found on the lower incisors, yet in all cases this 'ideal' etch was found to occupy less than $5 \%$ of the etched buccal surface enamel. The greatest area of etched enamel surface was occupied by Type C (etched but enamel prisms not evident). The mean bond strength values varied significantly between different tooth types, with the lowest bond strength found on the upper first molar (6.5 MPa) and the highest on the lower first molar (13.1 MPa). Conclusions: An ideal etch pattern is not essential in order to produce a strong bond.
\end{abstract}

Since Buonocore's ${ }^{1}$ finding in 1955 that acid etching of the enamel increases the bond strength of resin to enamel, the practice of bonding to enamel has become a routine procedure in many fields of dentistry.

One area that has received little attention has been the effect of variations in the enamel surface structure upon the bonding characteristics. Over the years, it has become accepted anecdotally

${ }^{1 *}$ Child Dental Health, ${ }^{2}$ Restorative Dentistry, Newcastle Dental School, Newcastle upon Tyne. ${ }^{*}$ Correspondence to: R.S. Hobson, Child Dental Health, Newcastle Dental School, Newcastle upon Tyne, England NE2 4BW.

Email:R.S.Hobson@ncl.ac.uk

\section{Refereed paper}

Received 26.02.01; Accepted 14.11.01

๑ British Dental Journal 2002; 192: 463-468 that a well-developed enamel etch pattern is a requirement for good bonding. There appears to be little evidence to justify this assumption.

Poole and Johnson ${ }^{2}$ were the first to classify enamel etch patterns, followed by Silverstone et $a .^{3}$ and more recently Brannstrom et $a .^{4}$ and Galil and Wright. ${ }^{5}$ They all reported that there are variations in both the quality and quantity of etched enamel on the tooth surface. Gwinnett ${ }^{6}$ and Whittaker ${ }^{7}$ proposed that the presence of aprismatic enamel may limit the development of the etch pattern; the resulting reduction in resin penetration contributing to a weaker bond strength, although the study did not demonstrate this effect.

It has been previously reported ${ }^{8}$ that an ideal etch pattern occupies less than 5\% of the etched enamel on the buccal surface of human teeth and that there are significant differences in the nature and extent of etch patterns for different tooth types. The present study was undertaken to investigate the structure of the etched enamel surface of different teeth and to examine the potential relationship between bond strength and the nature of the etched surface enamel.

\section{MATERIALS AND METHODS}

Twenty-eight patients, 10 male and 18 female between the ages of 11 and 16 years, with lifelong residence in Tyne and Wear, who were about to commence fixed orthodontic treatment, were recruited into the study. Following pumice cleansing and etching with 37\% phosphoric acid for 30 seconds, high resolution silicone impressions of the etched buccal surfaces of their teeth were recorded. 9,10

The technique has been shown to give a resolution of $0.2 \mu \mathrm{m}$, which is significantly smaller than the average size of an enamel prism end which is $4-5 \mu \mathrm{mm}$ across. ${ }^{9,10}$ The impressions were replicated using Spurrs' epoxy resin, gold coated and examined in the scanning electron microscope (SEM) at x1070 magnification with a long focal length to avoid distortion.

Histometry was employed to assess the extent and quality of etch patterns on the area used to bond orthodontic brackets. This area, which we have called the orthodontic bonding area (OBA), is well defined as the majority of brackets currently used in orthodontics are of the pre-adjusted type, which require accurate placement in the centre of the buccal surface of the crown. Bracket bases for different teeth have similar dimensions to each other, of approximately $4 \mathrm{~mm}$ square, and are bonded centrally on the mid point of the crown, this represents the area where bonding occurs - the OBA. 
Table 1 Mean percentage area occupied by each etch type (Type A, Type B, Type C, Type D) for upper and lower teeth

\begin{tabular}{|c|c|c|c|c|c|c|c|c|c|c|}
\hline \multirow[t]{3}{*}{ Tooth } & \multicolumn{10}{|c|}{ Etch type } \\
\hline & \multicolumn{5}{|c|}{ Lower } & \multicolumn{5}{|c|}{ Upper } \\
\hline & $n$ & A & B & C & D & $n$ & A & B & C & D \\
\hline Central incisor & 31 & 3.6 & 23.3 & 37.2 & 35.8 & 36 & 4.4 & 22.4 & 41.9 & 31.3 \\
\hline Lateral incisor & 25 & 1.6 & 11.4 & 39.8 & 47.3 & 28 & 4.2 & 14.4 & 38.1 & 43.4 \\
\hline Canine & 25 & 1.9 & 11.4 & 40.1 & 46.6 & 26 & 1.9 & 12.9 & 37.5 & 47.5 \\
\hline 1st Premolar & 6 & 1.3 & 6.7 & 33.9 & 58.1 & 13 & 0.5 & 6.1 & 38.7 & 54.8 \\
\hline 2nd Premolar & 20 & 3.3 & 13.2 & 45.2 & 38.3 & 14 & 3.1 & 23.6 & 36.4 & 36.9 \\
\hline 1st molar & 24 & 0.9 & 5.7 & 34.5 & 58.8 & 26 & 1.1 & 10.6 & 38.9 & 49.4 \\
\hline
\end{tabular}

The nature and quality of etching was assessed at regular intervals over the OBA. A grid with 30 intersections was overlaid on the SEM monitor and the etch pattern assessed at each intersection for that particular field. The field of observation was then moved $100 \mu \mathrm{m}$ to the right using the SEM vernier adjustment and the next field assessed. After eight fields were examined horizontally, the field of observation was moved down $100 \mu \mathrm{m}$ and the next series of fields examined at $100 \mu \mathrm{m}$ intervals as before. This was repeated in a grid pattern to give 1920 intersections. The method has been shown to give 95\% confidence in the accuracy of the data collected and is highly reproducible. ${ }^{10}$

A four-point scale ${ }^{10}$ was used to assess the quality of etch: Type A - well-developed 'conventional' etch pattern, with welldefined prisms; Type B - prisms apparent but poorly defined; Type C - no prism definition, but surface roughening has occurred; Type D - no discernible change in enamel surface, ie apparently no etching has occurred.

In order to examine the relationship between etch pattern and bond strength, 240 extracted teeth, 20 of each tooth type, were collected and A Company pre-adjusted orthodontic brackets bonded in their correct position (on the OBA) using Transbond lightcured orthodontic adhesive according to the manufacturer's instructions. All bonds and subsequent tests were undertaken within six months of extraction.

Twenty-four-hour bond strength testing was undertaken using an Instron testing machine, with a crosshead speed of $1 \mathrm{~mm} /$ minute, as described by Fox et al., ${ }^{11}$ and the results recorded in units of force (Newtons).

In addition, the Adhesive Remnant Index (ARI) ${ }^{12,13}$ was recorded for the tooth and bracket surfaces of all bonds tested.

All data were entered into Minitab 12.0 spreadsheets for statistical analysis using ANOVA, with post hoc Tukey tests. Linear regression was used to examine the relationships between etch pattern and bond strength. The ARI data were examined using a Kruskal-Wallis test.

\section{RESULTS}

The type of etch was not significantly affected by the side (left or right) of the mouth assessed ( $p=0.532)$. Data from the two sides were therefore combined. Overall, there was no significant difference between etch types for upper and lower teeth $(p=0.218)$; however, there were significant differences between specific teeth in the upper and lower arch as described below.

The mean percentage values of the area occupied by each etch pattern on each tooth type are given in Table 1. There was a general trend of an increase in apparently no etch (Type D) from anterior to posterior teeth with a trend of reducing well-defined etch (Types A and B) in the same direction. For etch Types A, B and D there was a significant difference between tooth types $(p<0.01)$. There was no apparent difference in the area occupied by etch Type $\mathrm{C}$ between teeth $(p=0.975)$.
The greatest amount of Type A etch (well-defined etched enamel prisms) was found on lower incisors, yet in all cases this 'ideal' etch was found to occupy less than 5\% of the etched enamel surface. The greatest area of etched enamel surface was occupied by Type C (etched but enamel prisms not evident), and it is also noteworthy that large areas of the enamel surface were recorded as unetched (Type D). If etch Types A and B are considered as the best for bonding, then it can be seen that these together occupy a small area of the etched buccal surface.

The mean bond strength values, along with their standard deviations, are given in Table 2 in units of force (Newtons), force per unit bracket width (Newtons/mm) and stress (Megapascals) and the Weibull parameters - characteristic strength and modulus - which were obtained by curve fitting (Fig P, Biosoft, Cambridge, UK).

When $n$ does not equal 20, this is due to the tooth moving within the acrylic mount block before debonding occurred.

The unit of force (Newton) is not an entirely appropriate means of expressing data from the bond strength studies as it does not allow for the variation in bracket size between tooth types. Using peel force $(\mathrm{N} / \mathrm{mm})$ allows for the fact that the method of debonding as described by Fox et al. ${ }^{11}$ has an element of peel and is not a true shear test, for which the results would be presented in units of stress (MPa). It can be seen that the use of peel or stress does not affect the differences in the rank order between teeth and, as stress (MPa) is the most common unit used to express bond strength, it was used for further data analysis.

The distributions of the Adhesive Remnant Index (ARI) and the number of bonds that failed due to enamel fracture are given in Tables 3 and 4. Altogether, 229 results were recorded; a small number of missing results occurred due to the tooth pulling out of the retaining acrylic block.

Analysis of variance showed that tooth type had a significant effect on mean bond strength $(p=0.0001)$; there being a twofold difference between the highest and lowest mean bond strength values (Table 2). The greatest mean bond strength was found on the lower first molars and the lowest on the upper first molars. In the upper arch, the anterior teeth (incisors and canines) exhibit greater bond strengths than the posterior teeth (premolars and molars) ( $p=0.0001)$. In the lower arch, the opposite was found the anterior teeth giving weaker bond strengths than the posterior teeth $(\mathrm{p}=0.001)$.

In the upper arch, the first molars exhibited significantly ( $p<0.05$ ) weaker bond strength than all the other upper teeth except for the second premolars. The lateral incisors gave significantly stronger bonds $(\mathrm{p}<0.05)$ than the first and second premolars and the first molar.

In the lower arch, first molars gave significantly stronger bonds $(\mathrm{p}<0.05)$ than the lateral incisors, the canines and the first premolar.

Student $t$-tests were undertaken to examine the differences in bond strength between equivalent teeth from upper and lower arches. Overall, there was no significant difference in the mean 
Table 2 Summary of mean 24-hour bond strength (force, force/linear width and stress) for different tooth types, with $95 \%$ Confidence Intervals, Weibull strength and modulus

\begin{tabular}{|c|c|c|c|c|c|c|c|c|c|}
\hline \multirow[t]{2}{*}{ Tooth Type } & \multirow[t]{2}{*}{$n$} & \multicolumn{2}{|c|}{ Force (Newtons) } & \multicolumn{2}{|c|}{$\begin{array}{l}\text { Force/linear bracket } \\
\text { width (Newton/mm) }\end{array}$} & \multicolumn{2}{|c|}{ Bond strength (MPa) } & \multirow[t]{2}{*}{$\begin{array}{l}\text { Weibull } \\
\text { strength (MPa) }\end{array}$} & \multirow[t]{2}{*}{$\begin{array}{l}\text { Weibull } \\
\text { modulus }\end{array}$} \\
\hline & & Mean & 95\% C.I. & Mean & 95\% C.I. & Mean & 95\% C.I. & & \\
\hline U1 - Upper central incisor & 20 & 190.7 & $163.4,218.0$ & 42.5 & $35.7,49.3$ & 11.6 & $8.6,14.6$ & 13.2 & 3.8 \\
\hline U2 - Upper lateral incisor & 20 & 161.9 & $147.2,176.6$ & 46.5 & $41.4,51.6$ & 12.6 & $10.6,14.6$ & 11.4 & 4.6 \\
\hline U3 - Upper canine & 20 & 175.7 & $162.8,188.6$ & 40.8 & $36.0,45.6$ & 11.0 & $8.6,13.4$ & 13.6 & 6.8 \\
\hline U4 - Upper first premolar & 20 & 128.6 & $110.8,146.4$ & 35.4 & $29.8,41.0$ & 9.6 & $7.1,12.1$ & 10.0 & 2.6 \\
\hline U5 - Upper second premolar & 19 & 119.2 & $101.3,137.1$ & 32.8 & $28.4,37.2$ & 8.9 & $6.5,11.3$ & 11.9 & 4.4 \\
\hline U6 - Upper first molar & 17 & 158.7 & $137.0,180.4$ & 26.4 & $21.0,31.8$ & 6.5 & $3.1,9.9$ & 10.1 & 2.3 \\
\hline L1 - Lower central incisor & 19 & 130.1 & $113.1,147.1$ & 37.7 & $32.7,42.7$ & 10.3 & $7.7,12.9$ & 10.8 & 3.4 \\
\hline L2 - Lower lateral incisor & 19 & 110.8 & $89.8,131.8$ & 32.1 & $26.5,37.7$ & 8.8 & $6.0,11.6$ & 10.7 & 3.6 \\
\hline L3 - Lower canine & 20 & 115.1 & $100.2,130.0$ & 31.9 & $27.0,36.8$ & 8.6 & $5.3,11.9$ & 9.8 & 3.6 \\
\hline L4 - Lower first premolar & 18 & 120.6 & $101.1,140.1$ & 33.2 & $28.0,38.4$ & 9.0 & $5.9,12.1$ & 12.7 & 4.1 \\
\hline L5 - Lower second premolar & 19 & 154.7 & $111.7,197.7$ & 42.6 & $37.8,47.4$ & 11.6 & $8.5,14.7$ & 8.1 & 1.4 \\
\hline L6 - Lower first molar & 17 & 296.7 & $254.1,339.3$ & 48.6 & $44.2,53.0$ & 13.1 & $9.2,17.0$ & 18.6 & 4.4 \\
\hline
\end{tabular}

Table 3 Number of teeth per Adhesive Remnant Index (ARI) score and number of teeth with enamel fractures for different tooth types.

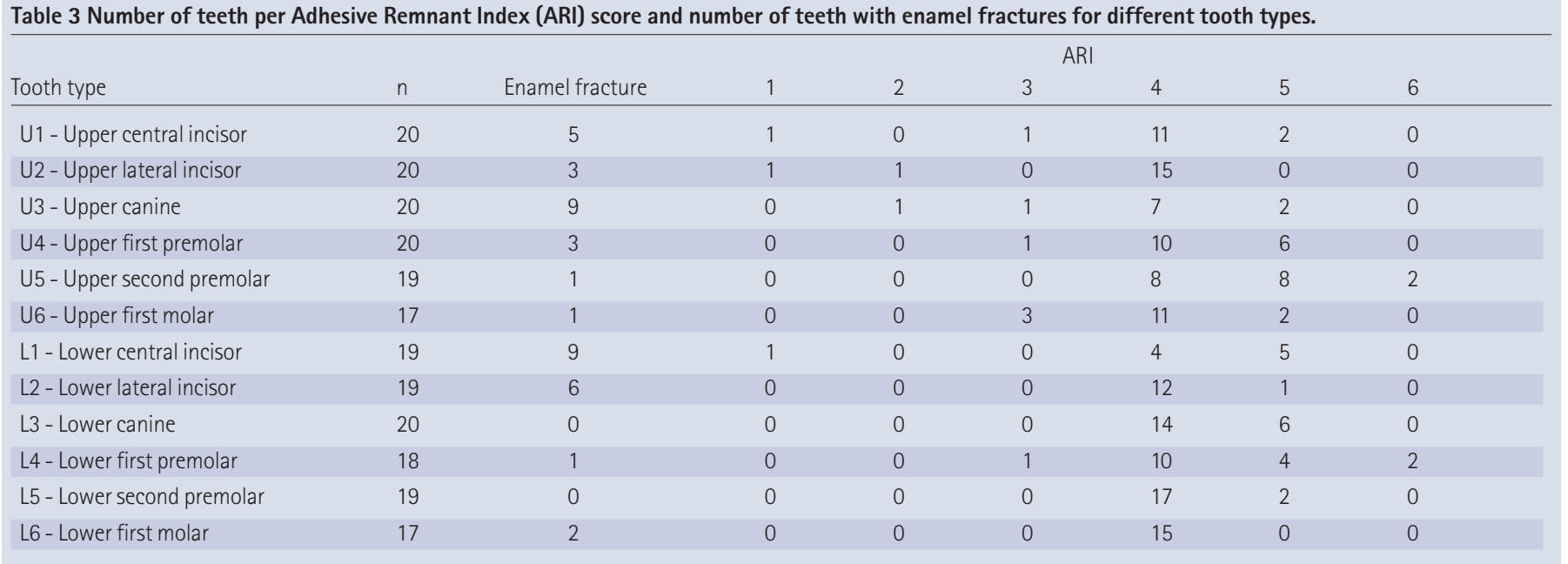

bond strength between teeth from the upper arch $(9.8 \mathrm{MPa})$ and teeth from the lower arch $(9.7 \mathrm{MPa})(\mathrm{p}=0.85)$. Also, there was no significant difference in bond strength between upper and lower central incisors $(\mathrm{p}=0.11)$ and upper and lower first premolars $(p=0.21)$. However, there were significant differences in bond strength when bonds between upper and lower lateral incisors $(p=0.0001)$, canines $(p=0.017)$, second premolars $(p=0.031)$ and first molars $(p=0.00001)$ were compared.

Bond strength data were analyzed in more detail using Weibull analysis. The characteristic Weibull strength (MPa) and the Weibull modulus is given in Table 2. Whereas in a gaussian distribution, a large standard deviation equates to a broad distribution, a large Weibull modulus indicates a narrow distribution.

It can be seen that the results for Weibull analysis show a similar overall trend to those using a normal distribution. The Weibull analysis was used to indicate probability of failure as a function of stress. Figures 1 and 2 show the Weibull distribution plots for different tooth types.

It can be seen that, in the upper arch, the upper first molar has the greatest probability of failure at a given stress, whereas in the lower arch, the first molar has the least probability of failure for any given stress.

Tooth type was found to have a significant effect on the ARI $(p=0.0001)$ (Table 3). The most frequent type of failure was associated with an ARI score of 4. Furthermore, upper teeth with the highest bond strengths (incisors and canines) suffered more enamel fractures than those with lower bond strengths. In the lower arch, the incisors suffered the greatest number of enamel fractures.
The median ARI scores for tooth and bracket surfaces as well as summed ARI scores are given in Table 4. It can be seen that the majority of bond failures were adhesive in nature, occurring at the bracket-composite interface, the majority of composite remaining on the tooth surface.

In order to examine the relationship between bond strength and etch pattern, regression analysis, using tooth type as the response variable, was undertaken.

Table 4 Median Adhesive Remnant Index values for tooth and bracket surfaces, and summed scores

\begin{tabular}{lllll}
\hline Tooth type & $n$ & $\begin{array}{l}\text { Tooth } \\
\text { surface }\end{array}$ & $\begin{array}{l}\text { Bracket } \\
\text { surface }\end{array}$ & $\begin{array}{l}\text { ARI } \\
\text { Summed }\end{array}$ \\
\hline U1 - Upper central incisor & 20 & 3 & 1 & 4 \\
U2 - Upper lateral incisor & 20 & 3 & 1 & 4 \\
U3 - Upper canine & 20 & 2 & 3 & 4 \\
U4 - Upper first premolar & 19 & 3 & 1 & 4 \\
U5 - Upper second premolar & 19 & 3 & 2 & 4 \\
U6 - Upper first molar & 18 & 1 & 3 & 4 \\
L1 - Lower central incisor & 20 & 2 & 1 & 5 \\
L2 - Lower lateral incisor & 20 & 3 & 1 & 4 \\
L3 - Lower canine & 19 & 3 & 1 & 4 \\
L4 - Lower first premolar & 19 & 3 & 2 & 4 \\
L5 - Lower second premolar & 18 & 3 & 1 & 4 \\
L6 - Lower first molar & 17 & 3 & 1 & 4
\end{tabular}




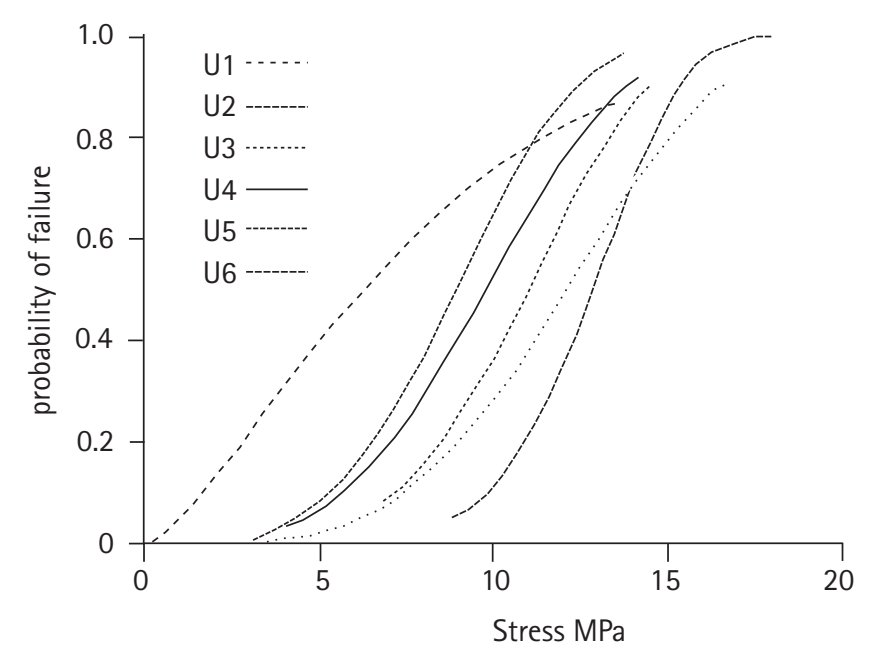

Fig. 1 Weibull distribution plot for probability of failure against stress (MPa) on upper teeth. U1 = Central incisor; U2 = Lateral incisor; U3 = Canine; U4 = First premolar; U5 = Second premolar; U6 = First molar.

The result of this analysis of the relationship between the proportion of etch Type A and bond strength is shown in Figure 3: there was no significant relationship between the two factors $(p=0.504)$. However, the lower first molar appeared to be lying well out - with the trend that bond strength was associated with a decreasing amount of Type A etch pattern. When the analysis was repeated excluding the lower first molar (Figure 4), a statistical significant relationship ( $p=0.015)$ appears between mean bond strength and percentage area occupied by etch Type A.

There were no significant relationships between other etch types (etch Type B, Type C and Type D) and mean bond strength $(p>0.05)$. There was also no relationship $(p=0.90)$ between bond strength and a combination of etch Types A and B, which together could intuitively be considered as desirable etch patterns for promoting increased bond strength.

\section{DISCUSSION}

The current work represents the first occasion on which data on bond strength and etch patterns has been collected in a single study for the purpose of attempting to correlate bond strength with quality and quantity of enamel etch development. This provides a unique opportunity to investigate a fundamental principle used in current clinical practice.

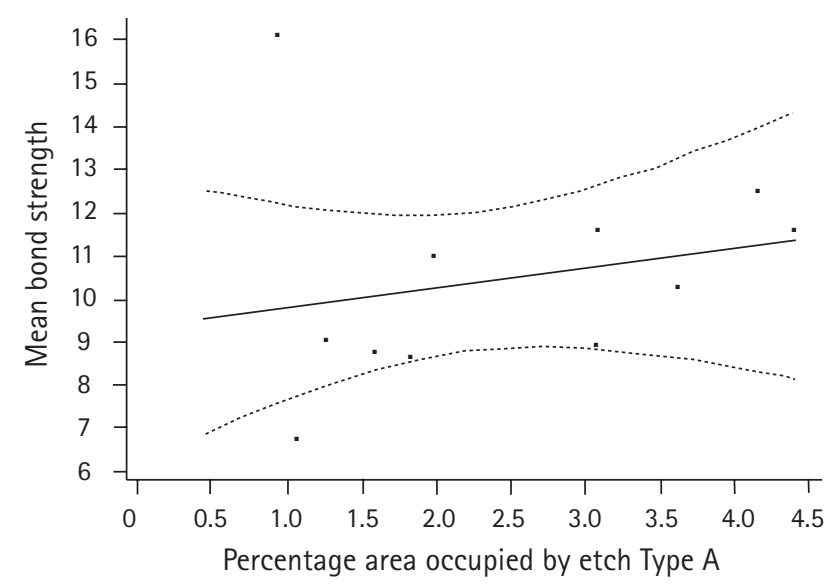

Fig. 3 Regression plot of mean bond strength (MPa) for 12 tooth types against percentage area of OBA occupied by Type $A$ (ideal) etch pattern. The solid line shows the regression line and the dotted lines the $95 \%$ confidence limits for the regression.

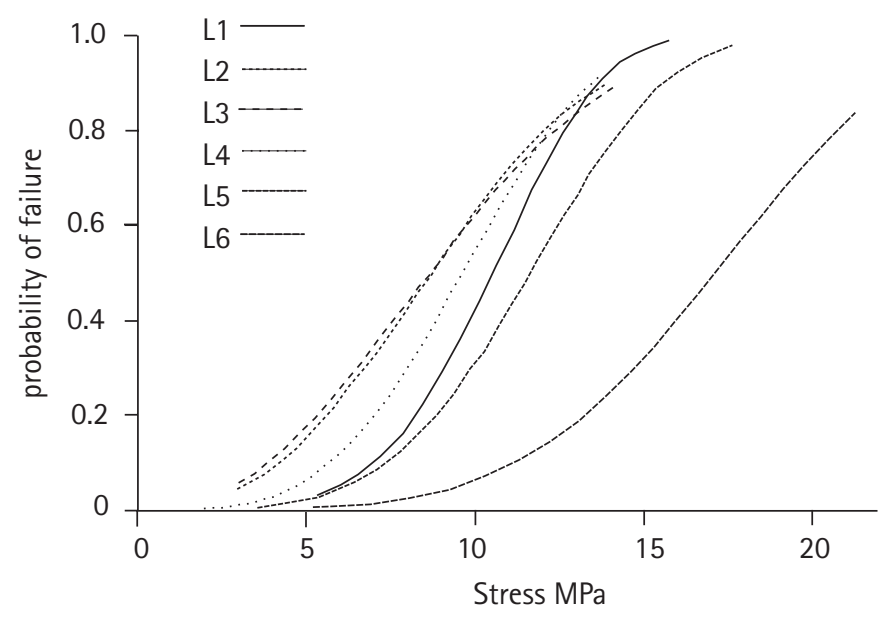

Fig. 2 Weibull distribution plot for probability of failure against stress (MPa) on lower teeth. L1 = Central incisor; L2 = Lateral incisor; L3 = Canine; L4 = First premolar; L5 = Second premolar; L6 = First molar. Lower first molars were further to the right, showing the least likelihood of failure at a given stress than other teeth. The lower second premolar, central incisor and first premolar showed an increase in probability of failure with stress. The lower canine and lateral incisor together show the greatest likelihood of failure at a given stress.

There was a significant difference in the mean bond strengths for different tooth types. The greatest mean bond strength was found on the lower first molars and the weakest on the upper first molars. ARI scores showed that the most common method of failure was adhesive (ARI 4), with lower incisors having a high level of enamel fracture.

The findings for bond strength between brackets and teeth in the upper arch agree well with the prediction of Whittaker ${ }^{7}$ that the increase in aprismatic enamel on posterior teeth may have an effect on bond strength. They are also consistent with clinical findings $^{14,15,16,17}$ that the bond failure rate is higher with posterior teeth, than anterior teeth.

In the lower arch, the opposite trend was found with greatest bond strength being achieved on the lower first molars. The Weibull curves confirm that the upper first molar had the weakest bond strength and the lower first molar had the highest.

This is surprising as the lower first molar is reported to have the greatest rate of bond failure in the clinical situation. ${ }^{14,15,16,17}$ The reason for the higher rate of bond failure for these teeth may be due to greater masticatory forces. ${ }^{16}$ If the stresses placed upon ortho-

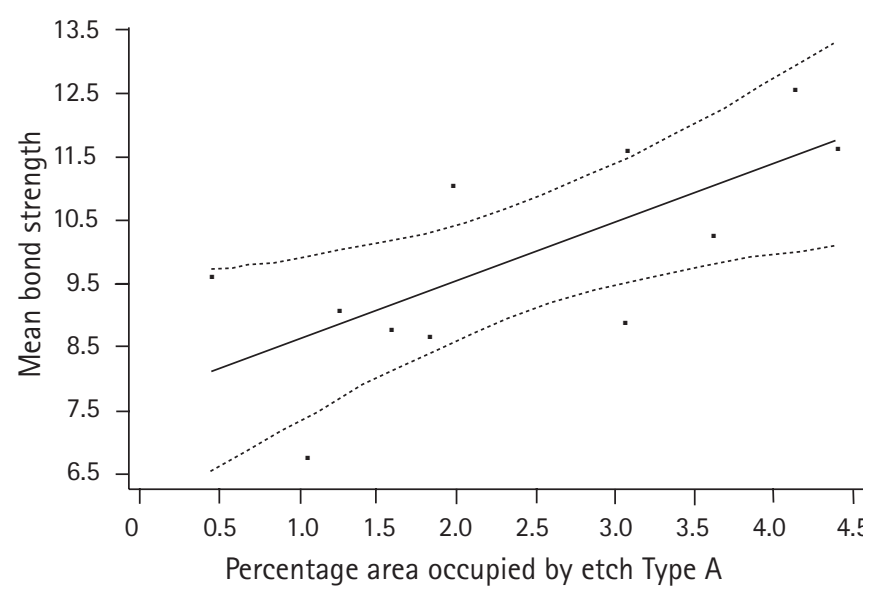

Fig. 4 Regression plot of mean bond strength (MPa) for 11 tooth types against percentage area of OBA occupied by Type $A$ (ideal) etch pattern (data for the lower first molar are excluded). The solid line shows the regression line and the dotted lines the $95 \%$ confidence limits for the regression. 
dontic brackets in the mouth were known, Weibull graphs could make it possible to estimate the predicted failure of a bond at known stress on different teeth.

Equally, it would be possible to estimate the potential stress experienced by an orthodontic bond from its failure rate. For example, if 20\% of orthodontic bonds on upper first molars failed in clinical practice, the Weibull curves (Figure 1) would predict a bond strength of $2.5 \mathrm{MPa}$ equating to this level of failure. This is an area for future research.

For the first premolars, bond strengths achieved with upper and lower teeth are of similar magnitude, but significant differences were found in the bond strength when upper and lower second premolars were compared. These results suggest that it would be unwise to use different types of teeth in surface enamel bond strength studies, unless the teeth in different groups are properly stratified.

The ARI showed that tooth type had a significant effect on mode failure, with the site of bond failure differing between tooth types. The most frequent mode of failure was adhesive failure at the composite-bracket interface (ARI score of 4). In the laboratory study, fracture occurred predominantly at the composite-bracket interface leaving the majority of residue composite on the tooth surface. This would indicate that bond strength is greatest at the enamel-composite interface. Fox et al. ${ }^{11}$ suggested that it would be preferable for bonds to fail at the enamel-composite interface as this would reduce the need for removal of composite from the tooth surface at debond following completion of fixed appliance treatment. However, if the bond between bracket and composite is greater than that between composite and enamel, there is an increased possibility of enamel fracture occurring during debond. This may explain some of the problems associated with iatrogenic enamel damage upon debonding of brackets in vivo. The present results suggest that a bonding regime that produces an adequate bond strength on premolars may result in an excessive bond strength for other teeth. Clinically, the number of enamel fractures that occur on debonding is a concern as it is normally anterior teeth that are affected. The results of this study suggest that, for incisors, a bond strength greater than 8-9 MPa more than exceeds the clinical requirement, particularly in respect of lower incisors.

The relationship between acid etch pattern and bond strength was conducted on the equivalent teeth. Because the two properties were not determined using the same teeth, the outcomes should be treated with some caution, despite the fact that the method of regression analysis is commonly used to investigate and model the relationship between a response variable and one or more predictors, which may be unconnected. It allows data from different sources to be examined for a possible relationship; in this case between acid etch pattern and bond strength.

In this study, bond failure is determined using the application of force at a specific time after bond placement. In the mouth, bond failure may be the result of progressive damage to the bond over a long time period due to multiple applications of load until failure occurs, ie by fatigue. The ball mill ${ }^{18,19}$ has been used to attempt to reproduce the cyclic loading of brackets thought to be the cause of failure in the mouth. However, as yet this laboratory method has not been fully validated against a clinical trial.

The findings of the current study suggest that the development of a well-defined 'ideal' etch pattern is not necessarily a pre-requisite for high-bond strength and that the relationship between etch pattern and bond strength is complex. Whittaker ${ }^{7}$ found that the extent of aprismatic enamel increased on posterior teeth in comparison with anterior teeth and he proposed that this may affect the acid etch procedure prior to bonding and hence bond strength and survival. A number of authors have supported this view that aprismatic enamel on the buccal surface may affect bond failure. ${ }^{20,21,22}$
One curious finding of the current study that is difficult to explain is the discrepancy between the poor quality of etch on the lower first molar and its large bond strength and vice versa with the upper first molar. Published work ${ }^{6,7,18}$ indicates that the etch pattern is poorly defined on lower first molars and therefore that bond strength should be low. However, in the current study it was found to be significantly higher than for any other tooth, suggesting that the quality of etch does not influence bond strength. This is further confirmed by the regression analysis, which indicates no significant relationship between bond strength and etch pattern. Only when the lower first molar is removed from the analysis does the relationship between etch pattern and bond strength become significant.

The traditional view of the mode of action of acid etching of enamel is that it causes surface roughening or porosity into which resins can penetrate. ${ }^{23}$ The outcome of the current study appears to support Nakabayashi's proposal ${ }^{24,25,26}$ that exposure of enamel crystallites (ie surface roughening) is more important than welldefined etch patterns. These workers suggested that the resin-enamel bond strength is the result of the cumulative crosssectional area of resin infiltrating the enamel space. The current work suggests that tag formation may not be as important as previously thought.

There is now some evidence that an enamel 'hybrid layer' may be involved in bonding, similar to that identified at the dentine-adhesive interface. ${ }^{27}$ Hybridisation is a process routinely used to achieve bonding to dentine by creating a mixture of adhesive polymers and dental hard tissues at a molecular-level. ${ }^{26}$ These authors have shown that unfilled resin can penetrate etched enamel so well that it encapsulates apatite crystals - thus, adhesion between resin and etched enamel may be achieved by hybrid layer formation.

This hybridisation theory can be used to explain the outcome of the current study and is further supported by the work of Leger et $a .^{28,29}$ who reported a poor relationship between depth of resin penetration in etched enamel and the resulting resin-enamel bond strength, suggesting that enamel porosity is more important than a well-defined etch pattern.

Although the present study sheds some light on the relationship between acid etching and bond strength, it is apparent that it is still somewhat unclear, and further investigation is required in order to determine the degree of etching required to produce adequate bond strength whilst minimizing enamel damage on debonding.

1. Buonocore M G. A simple method of increasing the adhesion of acrylic filling materials to enamel surfaces. J Dent Res $1955 ; \mathbf{3 4}: 849-853$.

2. Poole D F G, Johnson N W. The effects of different demineralising agents on human enamel as observed by electron microscopy. Arch Oral Biol 1967: 12: 1621-1634.

3. Silverstone L M, Saxton C A, Dogon I L, Fejerskov 0. Variation in the pattern of acid etching of human dental enamel examined by scanning electron microscopy. Caries Res 1975; 9: 373-387.

4. Brannstrom M, Nordenvall K J, Malmgren 0 . The effect of various pretreatment methods of the enamel in bonding procedures. Am J Orthod 1978; 74: 522-530.

5. Galil K A, Wright G Z. Acid etching patterns on buccal surfaces of permanent teeth. Pediatr Dent 1979: 1: 230-234.

6. Gwinnett A J. Human prismless enamel and its influence on sealant penetration. Arch Oral Biol 1973; 18: 441-444.

7. Whittaker D K. Structural variations in the surface zone of human tooth enamel observed by scanning electron microscopy. Arch Oral Biol 1982: 27: 383-392.

8. Mattick CR, Hobson R S. Variability in quality and quantity of etch pattern on different tooth types. J Dent Res 1997; 76: 1029 Abst 81.

9. Beynon A D. Replication technique for studying microstructure in fossil enamel. Scanning Microsc 1987; 1: 663-669.

10. Hobson R S, Mattick CR, Parker A G. The validation of a technique for replication of acid etch patterns in vivo. J Dent Res 1996; 75: 1173 Abst 351.

11. Fox N A, McCabe J F, Buckley J G. A critique of bond strength testing in orthodontics. BrJ Orthod 1994; 21:33-43.

12. Artun J, Bergland S. Clinical trials with crystal growth conditioning as an alternative to acid etch enamel pretreatment. Am J Orthod 1984: 85:333-40.

13. Larmour C J, McCabe J F, Gordon P H. An ex-vivo investigation into the effects of chemical solvents on the debond behaviour of ceramic orthodontic brackets. $\mathrm{Br} J$ Orthod 1998; 25: 35-39. 
14. Zachrisson B J. A post treatment evaluation of direct bonding in orthodontics. Am J Orthod 1977; 71: 173-189.

15. Newman G V. A post treatment survey of direct bonding of metal brackets. Am J Orthod 1978; 74: 197-206.

16. Gorelick L, Geiger A M, Gwinnett A J. Implications of the failure rates of bonded brackets and eyelets: a clinical study. Am J Orthod 1984; 86: 403-406.

17. Kinch A P, Taylor H, Warltier R, Oliver R G, Newcombe R G. A clinical trial comparing the failure rates of directly bonded brackets using etch times of 15 or 60 seconds. Am J Orthod 1988; 94: 476-483.

18. Millett D T, McCabe J F, Bennett T G, Carter N E, Gordon P H. The effect of sandblasting on the retention of first molar orthodontic bands cemented with glass ionomer cement. BrJ Orthod 1995; 22: 161-169.

19. Abu Kasim N H, Millett D T, McCabe J F. The ball mill as a means of investigating the mechanical failure of dental materials. J Dent 1996; 24: 117-124.

20. Millett D T, Gordon P H. A 5-year clinical review of bond failure with a no-mix adhesive (Right on). Eur J Orthod 1994; 16: 203-211.

21. Sunna S, Rock W P. Clinical performance of orthodontic brackets and adhesive systems: a randomized clinical trial. Br J Orthod 1998; 25: 283-287.

22. Sunna S, Rock P. An ex vivo investigation into the bond strength of orthodontic brackets and adhesive systems. Br J Orthod 1999; 26: 47-50.

23. Buonocore M G, Matsui $A$, Gwinnett. A J. Penetration of resin dental materials into enamel surfaces with reference to bonding. Arch Oral Biol 1968; 13:61-70.

24. Nakabayashi N, Takeyama M, Kojima K, Mogi M, Miura F, Masuhara E. Studies on dental self-curing resins (22) - adhesion of 4-META/MMA-TBB resin to enamel (author's transl)]." Shika Rikogaku Zasshi 1982; 23: 88-92.

25. Gunadi $G$, Nakabayashi N. Preparation of an effective light-cured bonding agent for orthodontic application. Dent Mater 1997; 13: 7-12.

26. Nakabayashi N, Pashley D H. Hybridization of dental hard tissues. Tokyo: Quintessence, 1998.

27. Hotta K, Mogi M, Miura F, Nakabayashi N. Effect of 4-MET on bond strength and penetration of monomers into enamel. Dent Mater 1992; 8: 173-5.

28. Legler L R, Retief D H, Bradley E L, Denys FR, Sadowsky P L. Effects of phosphoric acid concentration and etch duration on the shear bond strength of an orthodontic bonding resin to enamel. An in vitro study. Am J Orthod Dentofacial Orthop 1989; 96: 485-492.

29. Legler $L R$, Retief D H, Bradley E L. Effects of phosphoric acid concentration and etch duration on enamel depth of etch: an in vitro study. Am J Orthod Dentofacial Orthop 1990; 98: 154-160. 